\title{
Geometric approach to the Weil-Petersson symplectic form
}

\author{
Reynir Axelsson and Georg Schumacher
}

Dedicated to Professor Reinhold Remmert on the occasion of his 80th birthday

\begin{abstract}
In a family of compact, canonically polarized, complex manifolds the first variation of the lengths of closed geodesics is computed. As an application, we show the coincidence of the Fenchel-Nielsen and Weil-Petersson symplectic forms on the Teichmüller spaces of compact Riemann surfaces in a purely geometric way. The method can also be applied to situations like moduli spaces of weighted punctured Riemann surfaces, where the methods of Kleinian groups are not available.
\end{abstract}

Mathematics Subject Classification (2000). 32G15; 53C55, 53D30.

Keywords. Weil-Petersson form, Fenchel-Nielsen coordinates, symplectic geometry.

\section{Introduction}

In 1958 André Weil introduced the methods of deformation theory of compact complex manifolds to the study of Teichmüller spaces [WE]. In particular, he used the $L^{2}$-inner product, induced by a metric of constant negative curvature, to the harmonic representatives of Kodaira-Spencer classes. He conjectured that the resulting Hermitian inner product on the Teichmüller space would be of Kähler type and of negative curvature. The resulting Kähler form is since known as the Weil-Petersson form.

Beginning in 1982, Wolpert analyzed the Fenchel-Nielsen structure of the Teichmüller space and showed that the standard symplectic form $\omega^{\mathrm{FN}}=-\sum d \tau^{i} \wedge d \ell^{i}$, defined in terms of Fenchel-Nielsen coordinates, actually coincides with the WeilPetersson form $\omega^{\mathrm{WP}}$. This was achieved in a sequence of papers [WO2], [WO3], [WO4], which also contain important arithmetic results. Most of the arguments use in an essential way the theory of Kleinian groups. For example, one key idea in Wolpert's approach is to solve Beltrami equations on the upper half plane, to compute derivatives of cross-ratios of the solutions, and then use Poincaré series, in order to descend to the Riemann surfaces. 
Our aim is to extend these results to cases where the original methods do not apply, i.e., to cases where the hyperbolic structure is not necessarily induced by the universal covering, such as weighted punctured Riemann surfaces. Our methods also provide a purely differential geometric approach to a key result in Wolpert's approach, namely the Weil-Petersson duality of the twist vector fields $\partial / \partial \tau^{j}$ and the differentials $d \ell^{j}$ of the corresponding length functions. We use path integrals along closed geodesics of harmonic Beltrami differentials and holomorphic quadratic differentials. More generally, the first variation of the length of a closed geodesic in a holomorphic family of Kähler-Einstein manifolds of negative curvature can be expressed as the integral of the harmonic Kodaira-Spencer tensor along a geodesic; this is our Theorem 3.2.

We consider the Teichmüller space of (possibly weighted) punctured Riemann surfaces of genus $p$ with $n$ punctures. The existence of pants decompositions and Fenchel-Nielsen coordinates was shown by Zhang in $[\mathrm{ZH}]$ under the natural assumption that the cone angles do not exceed the value of $\pi$, i.e., the weights are in the interval $[1 / 2,1]$ (cf. Section 8). Let $s^{\kappa} ; \kappa=1, \ldots, N$ be local holomorphic coordinates on the Teichmüller space near a fixed point $s_{0}$ corresponding to a Riemann surface $X$; then the tangent vectors $\partial /\left.\partial s^{\kappa}\right|_{s_{0}}$ correspond to harmonic Beltrami differentials $\mu_{\kappa}$ on $X$. An immediate consequence of Theorem 3.2 is the following result:

Theorem 1.1. The complex derivative of a length coordinate with respect to a holomorphic coordinate equals half the integral of the corresponding harmonic Beltrami differential along the closed geodesic:

$$
\left.\frac{\partial \ell^{i}(s)}{\partial s^{\kappa}}\right|_{s_{0}}=\frac{1}{2} \int_{\gamma_{i}} \mu_{\kappa} .
$$

The above formula is independent of the choice of a set of Fenchel-Nielsen coordinates and holds for the length function $\ell^{\gamma}(s)$ of a differentiable family $\gamma(s)$ of closed geodesics.

In the classical case, closely related results, where the first variation of the length of a closed geodesic is expressed in terms of integrals, can be found in [WO1], [WO2].

The shearing technique for twists along closed geodesics plays an important role in Teichmüller theory. We handle the twist parameters $\tau^{i}$ in the context of deformation theory and cocycles of vertical automorphisms. This reflects only one aspect of the complex earthquake map associated to a given closed geodesic in the sense of McMullen [Mc] (cf. also [E-M]). The tangent vector $\partial /\left.\partial \tau^{i}\right|_{s_{0}}$ of the Teichmüller space is uniquely determined by its action on the cotangent space, i.e., the space of holomorphic quadratic differentials. (Here we use the type decomposition of the real tangent space of the Teichmüller space as embedded into its complexification.) 
Theorem 1.2. The tangent vector $\partial /\left.\partial \tau^{i}\right|_{s_{0}}$ applied to a quadratic holomorphic differential $\varphi=\varphi(z) d z^{2}$ is given by the integration of the quadratic differential along the corresponding closed geodesic:

$$
\left(\left.\frac{\partial}{\partial \tau^{i}}\right|_{s_{0}}, \varphi(z) d z^{2}\right)=\frac{\sqrt{-1}}{2} \int_{\gamma_{i}} \varphi .
$$

Note that also the above formula is independent of the choice of Fenchel-Nielsen coordinates. It holds for the twist vector $\partial / \partial \tau^{\gamma}$ for any given closed geodesic $\gamma$.

Observe that the above inner product is actually a product of the $(1,0)$-part of $\left(\partial / \partial \tau^{i}\right)$ and the quadratic holomorphic differential $\varphi$. As a corollary we obtain a key result proved by Wolpert for the classical case in [WO2].

Theorem 1.3. The Fenchel-Nielsen symplectic form on the Teichmüller space is equal to the Weil-Petersson form:

$$
\omega^{\mathrm{FN}}=\omega^{\mathrm{WP}}, \quad \text { i.e., }-\sum_{i=1}^{N} d \tau^{i} \wedge d \ell^{i}=\sqrt{-1} G_{\kappa \bar{\lambda}}^{\mathrm{WP}} d s^{\kappa} \wedge d s^{\bar{\lambda}} .
$$

In fact the formulas (1), (2) and (3) are intimately related in the sense that any two of them imply the third one.

In the situation of Fuchsian groups, the above geodesic integral (2) seems to be related to the monodromy integral of the quotient of the given quadratic differential by a certain Abelian differential, and a term involving the trace of the resp. hyperbolic group element in [WO2] (cf. Section 6).

Our approach can also be used to compute second variations - our computations and applications will appear elsewhere.

Acknowledgements. The second named author would like to thank Inkang Kim for stimulating discussions. The authors would like to thank the referee for pointing out the reference $[\mathrm{E}-\mathrm{M}]$.

This paper was written at the University of Iceland. The second named author would like to express his thanks for the kind hospitality and support.

\section{Families of Kähler-Einstein manifolds}

Let $\left\{\mathcal{X}_{s}\right\}_{s \in S}$ be a holomorphic family of canonically polarized compact complex manifolds parameterized by a (connected) complex space $S$. It is given by a proper, smooth, holomorphic mapping $f: \mathcal{X} \rightarrow S$ such that $\mathcal{X}_{s}=f^{-1}(s)$ for all $s \in S$. For simplicity we will assume that the base $S$ is smooth, although our results can also be given a meaning for possibly non-reduced singular base spaces. 
We denote by $X=\mathcal{X}_{s_{0}} ; s_{0} \in S$ a distinguished fiber. Let $n=\operatorname{dim}_{\mathbb{C}} X$, and denote by $z^{\alpha} ; \alpha=1, \ldots, n$ local holomorphic coordinates on $X$.

A Kähler form on $X$ will be denoted by

$$
\omega_{X}=\sqrt{-1} g_{\alpha \bar{\beta}} d z^{\alpha} \wedge d z^{\bar{\beta}} .
$$

On the fiber $X$ we are using the summation convention together with the $\nabla$-notation for covariant derivatives. A $\mid$-symbol will denote an ordinary derivative. Also, $\partial_{\alpha}$ and $\partial_{\bar{\beta}}$ will stand for $\partial / \partial z^{\alpha}$ and $\partial / \partial z^{\bar{\beta}}$ respectively. The raising and lowering of indices is defined as usual in terms of covariant derivatives.

For the Ricci tensor $R_{\alpha \bar{\beta}}$ of $X$ we will use the sign convention

$$
R_{\alpha \bar{\beta}}=-\log (g(z))_{\mid \alpha \bar{\beta}},
$$

where $g(z)=\operatorname{det}\left(g_{\alpha \bar{\beta}}(z)\right)$.

Accordingly we set $g(z, s)=\operatorname{det}\left(g_{\alpha \bar{\beta}}(z, s)\right)$ for the family $f: \mathcal{X} \rightarrow S$, where we equip the fibers $\mathcal{X}_{s}$ with Kähler-Einstein forms

$$
\omega \chi_{s}=\sqrt{-1} g_{\alpha \bar{\beta}}(z, s) d z^{\alpha} \wedge d z^{\bar{\beta}}
$$

of constant negative curvature -1 , i.e., $R_{\alpha \bar{\beta}}(z, s)=-g_{\alpha \bar{\beta}}(z, s)$.

Let

$$
\rho: T_{S_{0}} S \rightarrow H^{1}\left(X, \mathcal{T}_{X}\right)
$$

be the Kodaira-Spencer map for the corresponding deformation of $X$ over $S$ at the point $s_{0} \in S$.

A natural inner product on the space $H^{1}\left(X, \mathcal{T}_{X}\right)$ of infinitesimal deformations of $X$, the Weil-Petersson Hermitian inner product on $T_{s_{0}} S$, is induced by the KählerEinstein metric $\omega_{X}$ on $X$. Namely, given tangent vectors $u, v \in T_{s_{0}} S$, we denote by $A_{u}=A_{u \bar{\beta}}^{\alpha} \partial_{\alpha} d z^{\bar{\beta}}$ and $A_{v}$ the harmonic representatives of $\rho(u)$ and $\rho(v)$ respectively. Then the inner product of $u$ and $v$ equals

$$
\langle u, v\rangle_{\mathrm{WP}}=\int_{X} A_{u \bar{\beta}}^{\alpha} A_{\bar{v} \gamma}^{\bar{\delta}} g_{\alpha \bar{\delta}} g^{\bar{\beta} \gamma} g d V,
$$

where $A_{\bar{v}}$ denotes the adjoint (conjugate) tensor of $A_{v}$, and $g d V$ the volume element.

We note that the Weil-Petersson inner product is positive definite at a given point of the base, if the induced deformation is effective.

Let $s_{j} ; j=1, \ldots, N$ be local coordinates on $S$, and set $A_{j}=A_{\partial / \partial s_{j}}$. Then the Weil-Petersson form on $S$ equals

$$
\omega^{\mathrm{WP}}=\sqrt{-1} G_{i \bar{\jmath}}^{\mathrm{WP}}(s) d s^{i} \wedge d s^{\bar{j}},
$$


where we use the notation

$$
G_{i \bar{\jmath}}^{\mathrm{WP}}(s)=\left\langle\partial / \partial s^{i}, \partial / \partial s^{j}\right\rangle_{\mathrm{WP}}=\int_{X} A_{i \bar{\beta}}^{\alpha} A_{\bar{\jmath} \gamma}^{\bar{\delta}} g_{\alpha \bar{\delta}} g^{\bar{\beta} \gamma} g d V .
$$

A closed real $(1,1)$-form on the total space $\mathcal{X}$ of the given family is defined by

$$
\omega x=-\sqrt{-1} \partial \bar{\partial} \log g(z, s) .
$$

The Kähler-Einstein condition for the fibers implies that the restrictions of $\omega x$ to the fibers are the given Kähler-Einstein metrics:

$$
\omega x \mid x_{s}=\omega x_{s}
$$

for all $s \in S$.

An important fact is that the family of Kähler-Einstein metric tensors contains the harmonic representatives of Kodaira-Spencer classes. The short exact sequence

$$
0 \rightarrow \mathcal{T}_{X / S} \rightarrow \mathcal{T}_{X} \rightarrow f^{*} \mathcal{T}_{S} \rightarrow 0
$$

induces the Kodaira-Spencer map via the edge homomorphism for direct images. Again, we use $\nabla$-notation for covariant derivatives as well as raising and lowering of indices in fiber direction for families. A lift of a tangent vector $\partial / \partial s^{i}$ of $S$ at $s_{0}$ as a differentiable vector field of $\mathcal{X}$ on $X$ is of the form

$$
\partial / \partial s^{i}+b_{i}^{\alpha} \partial_{\alpha}
$$

Its exterior $\bar{\partial}$-derivative $B_{i \bar{\beta}}^{\alpha} \partial_{\alpha} d z^{\bar{\beta}}$, where $B_{i \bar{\beta}}^{\alpha}=\nabla_{\bar{\beta}} b_{i}^{\alpha}$, is interpreted as a $\bar{\partial}$-closed $(0,1)$-form on $X$ with values in the tangent bundle of $X$. It represents the obstruction against the existence of a holomorphic lift of the given tangent vector, i.e., the infinitesimal triviality of the deformation. Moreover,

$$
\rho\left(\partial / \partial s^{i}\right)=\left[B_{i \bar{\beta}}^{\alpha} \partial_{\alpha} d z^{\bar{\beta}}\right] \in H^{1}\left(X, \mathcal{T}_{X}\right) .
$$

Our argument is based upon the notion of canonical lifts in the sense of Siu (cf. [SIU]), which turned out to be $\omega x$-horizontal: The form $\omega x$ is positive definite in fiber directions but need not be positive on $\mathcal{X}$. Its components in the directions of $z^{\alpha}$ and $s^{j}$ are denoted by $g_{\alpha \bar{j}}$ etc. However, horizontal lifts of tangent vectors are well-defined and of the form

$$
\partial / \partial s^{i}+a_{i}^{\alpha} \partial_{\alpha}
$$

where

$$
a_{i}^{\alpha}=-g^{\bar{\beta} \alpha} g_{i \bar{\beta}} .
$$

We set $A_{i \bar{\beta}}^{\alpha}=\nabla_{\bar{\beta}} a_{i}^{\alpha}$ and obtain (cf. [SCH2], [SCH3]) the following fact: 
Proposition 2.1. The horizontal lifts with respect to $\omega x$ of tangent vectors induce harmonic representatives of Kodaira-Spencer classes: The harmonic representative of $\rho\left(\partial / \partial s^{i}\right)$ equals $A_{i \bar{\beta}}^{\alpha} \partial_{\alpha} d z^{\bar{\beta}}$. These satisfy the following properties:

(i) $\nabla_{\bar{\delta}} A_{i \bar{\beta}}^{\alpha}=\nabla_{\bar{\beta}} A_{i \bar{\delta}}^{\alpha}$,

(ii) $\nabla_{\gamma} A_{i \bar{\beta}}^{\alpha} g^{\bar{\beta} \gamma}=0$,

(iii) $A_{i \bar{\beta} \bar{\delta}}=A_{i \bar{\delta} \bar{\beta}}$.

The conditions (i) and (ii) above correspond to harmonicity, whereas condition (iii) reflects the relationship with the metric tensor.

\section{Families of closed geodesics}

Let $(f: \mathcal{X} \rightarrow S, \omega x)$ be a family of Kähler-Einstein manifolds with constant negative Ricci curvature equal to -1 , where $\omega x$ is given by (4).

We denote by $\gamma_{s}$ a differentiable family of closed geodesics contained in the fibers $X_{s}$. In order to describe the variation of the length of closed geodesics in a family, we use the notion of integrating a tensor along a geodesic. Exemplarily we define:

Definition 3.1. Let $C=C_{\bar{\beta} \bar{\delta}}$ be a tensor on the Kähler manifold $X$, and let $\gamma$ be a geodesic of length $\ell$, parameterized by $u(t)=\left(u^{1}(t), \ldots, u^{n}(t)\right)$, such that $\|\dot{u}(t)\|=1$. Then

$$
\int_{\gamma} C=\int_{\gamma} C_{\bar{\beta} \bar{\delta}} d z^{\bar{\beta}} d z^{\bar{\delta}}:=\int_{0}^{\ell} C_{\bar{\beta} \bar{\delta}}(u(t)) \dot{u}^{\bar{\beta}} \dot{u}^{\bar{\delta}} d t .
$$

For contravariant tensors of order one this notation coincides with the integration of a differential form along the curve $\gamma$.

The main result of this section is the following theorem:

Theorem 3.2. Let $\mathcal{X} \rightarrow S$ be a family of canonically polarized, compact, complex manifolds equipped with Kähler-Einstein metrics. Let $\gamma_{s}$ be a differentiable family of closed geodesics on the fibers $\mathcal{X}_{s}$, having the lengths $\ell(s)$. Let $\partial /\left.\partial s^{i}\right|_{s=s_{0}} \in T_{s_{0}} S$ be a tangent vector. Then the first variation of the length of the closed geodesic with respect to the holomorphic parameter of the family is half the integral of the symmetric, harmonic Kodaira-Spencer tensor along the geodesic:

$$
\left.\frac{\partial}{\partial s^{i}}\right|_{s=s_{0}} \ell(s)=\frac{1}{2} \int_{\gamma\left(s_{0}\right)} A_{i \bar{\beta} \bar{\delta}} d z^{\bar{\beta}} d z^{\bar{\delta}} .
$$


In order to prove the theorem, we may assume that $S=\{s\}$ is a disk in $\mathbb{C}$, and $s_{0}=0$. The closed geodesic curves $\gamma_{s}$ are solutions of the equation for geodesics

$$
\ddot{u}^{\alpha}(t, s)+\Gamma_{\gamma \sigma}^{\alpha}(u(t, s)) \dot{u}^{\gamma}(t, s) \dot{u}^{\sigma}(t, s)=0 .
$$

The solution is unique up to the constant value of the speed

$$
\|\dot{u}(t, s)\|^{2}=g_{\alpha \bar{\beta}}(u(t, s), s) \dot{u}^{\alpha}(t, s) \dot{u}^{\bar{\beta}}(t, s) .
$$

For $s=0$ we chose $\|\dot{u}\|=1$, for the remaining values of $s$ the value of $\|\dot{u}\|$ will be determined by the fact that the parameter $t$ assumes values in the interval $\left[0, \ell_{0}\right]$, where $\ell_{0}$ is the length of $\gamma_{0}$. Now

$$
\ell(s)=\int_{0}^{\ell_{0}}\|\dot{u}(t, s)\| d t
$$

so that

$$
\left.\frac{d \ell(s)}{d s}\right|_{s=0}=\frac{1}{2} \int_{0}^{\ell_{0}} \frac{d}{d s}\|\dot{u}(t, s)\|^{2} d t .
$$

We need to study various tensors on $\mathcal{X}$ along the geodesics. In particular, we consider the vector field

$$
U_{s}(t, s)=\left.\left(\frac{\partial}{\partial s}+u_{s}^{\alpha}(t, s) \frac{\partial}{\partial z^{\alpha}}+u_{s}^{\bar{\beta}}(t, s) \frac{\partial}{\partial z^{\bar{\beta}}}\right)\right|_{u(t, s)},
$$

where

The function

$$
u_{s}^{\alpha}(t, s)=\frac{\partial u^{\alpha}(t, s)}{\partial s} \quad \text { and } \quad u_{s}^{\bar{\beta}}(t, s)=\frac{\partial u^{\bar{\beta}}(t, s)}{\partial s} .
$$

$$
\chi(t, s)=\left\langle U_{s}, \dot{u}\right\rangle
$$

is defined as the inner product of vector fields along the geodesics with values on the total space $\mathcal{X}$. Concerning $\omega_{\mathcal{X}}$ we indicate the direction of the one-dimensional space $S$ by $s$. We have

$$
\chi(t, s)=g_{s \bar{\beta}} \dot{u}^{\bar{\beta}}+g_{\alpha \bar{\beta}} u_{s}^{\alpha} \dot{u}^{\bar{\beta}}+g_{\alpha \bar{\beta}} \dot{u}^{\alpha} u_{s}^{\bar{\beta}} .
$$

Using (8) we simplify the expression for the derivative $\dot{\chi}$ and get

$$
\begin{gathered}
\dot{\chi}(t, s)=g_{s \bar{\beta} \mid \alpha} \dot{u}^{\alpha} \dot{u}^{\bar{\beta}}+g_{s \bar{\beta} \mid \bar{\delta}} \dot{u}^{\bar{\beta}} \dot{u}^{\bar{\delta}}+g_{s \bar{\beta}} \ddot{u}^{\bar{\beta}}+g_{\alpha \bar{\beta} \mid \gamma} \dot{u}^{\gamma} u_{s}^{\alpha} \dot{u}^{\bar{\beta}} \\
+g_{\alpha \bar{\beta}} \dot{u}_{s}^{\alpha} \dot{u}^{\bar{\beta}}+g_{\alpha \bar{\beta} \mid \bar{\delta}} \dot{u}^{\alpha} u_{s}^{\bar{\beta}} \dot{u}^{\bar{\delta}}+g_{\alpha \bar{\beta}} \dot{u}^{\alpha} \dot{u}_{s}^{\bar{\beta}} .
\end{gathered}
$$

\section{Lemma 3.3.}

$$
\frac{\partial}{\partial s}\|\dot{u}\|^{2}-\dot{\chi}=A_{s \bar{\beta} \bar{\delta}} \dot{u}^{\bar{\beta}} \dot{u}^{\bar{\delta}}
$$


Proof. We use (8) and (10) together with (6) and (7) and apply Proposition 2.1. The statement now follows from a calculation.

The proof of Theorem 3.2 concludes with (9) and Lemma 3.3.

\section{Cocycles of vertical automorphisms}

Let $X$ be a compact complex manifold, and $\left(S, s_{0}\right)$ a germ of a complex space. We follow the approach of Forster and Knorr [F-K] and assign to any deformation of $X$ over a $\left(S, s_{0}\right)$ an element of the first cohomology $H^{1}\left(X, \mathcal{S}_{S}\right)$ of $X$ with values in the sheaf $G_{S}$ of vertical automorphisms. Its derivative with respect to the base space yields the Kodaira-Spencer map. For any deformation of $X$ given by a cartesian diagram

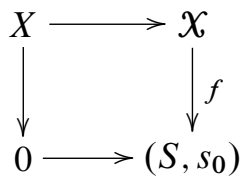

there exist open coverings $\left\{U_{i}\right\}$ of $X$ and $\left\{Z_{i}\right\}$ of $X$ together with isomorphisms $\psi_{i}: U_{i} \times S \rightarrow Z_{i}$, compatible with the cartesian diagram, and equal to the identity for $s=0$. The actual cocycles $\gamma_{i j}$ are defined on $\left(U_{i} \cap U_{j}\right) \times S$ by $\psi_{j}^{-1} \circ \psi_{i}$. We look at the derivatives of the $\gamma_{i j}$ with respect to tangent vectors of the base and values in the sheaf of holomorphic vector fields. These define the Kodaira-Spencer classes and will be compared with suitable $(0,1)$-forms representing the same classes.

We take a differentiable trivialization $\zeta: X \rightarrow X \times S$ of the family, which equals the identity for $s=s_{0}$. Then, according to (5), the class $\left.\rho\left(\partial / \partial s^{\kappa}\right)\right|_{s=s_{0}}$ is represented by

$$
\left.\frac{\partial^{2} \zeta^{\alpha}(z, s)}{\partial s^{\kappa} \partial z^{\bar{\beta}}}\right|_{s=s_{0}} \frac{\partial}{\partial z^{\alpha}} d z^{\bar{\beta}} .
$$

We now consider the situation where the covering that is involved in the deformation only consists of only two elements, $U_{1}$ and $U_{2}$, say. A differentiable trivialization of the holomorphic family can be chosen to be equal to the identity on the complement of any given compact neighborhood of $Z_{1} \cap Z_{2} \subset \mathcal{X}$.

\section{Application to Riemann surfaces}

For families of Riemann surfaces (with notation from Section 1) the above Proposition 2.1 states that harmonic Beltrami differentials are of the form

$$
\mu_{\kappa}=\mu_{\kappa}(z) \frac{\partial}{\partial z} \overline{d z}=-\frac{\partial}{\overline{\partial z}}\left(\frac{1}{g} \frac{\partial^{2} \log g}{\partial s^{\kappa} \overline{\partial z}}\right) \frac{\partial}{\partial z} \overline{d z} .
$$


We write these also in the form

$$
\mu_{\kappa}(z) \frac{\partial}{\partial z} \overline{d z}=\frac{\varphi_{\bar{\kappa}}(z)}{g(z)} \frac{\partial}{\partial z} \overline{d z}
$$

for certain quadratic holomorphic differentials $\varphi_{\kappa}=\varphi_{\kappa}(z) d z^{2}$, with $\varphi_{\bar{\kappa}}:=\overline{\varphi_{\kappa}}$.

Now Theorem 1.1 is an immediate consequence of Theorem 3.2.

\section{Twist coordinates vs. holomorphic coordinates}

In this section we will prove Theorem 1.2. We will first treat the case of Riemann surfaces $X$ equipped with the hyperbolic metrics $g=g(z)|d z|^{2}$. Given the context, we normalize a metric of constant curvature -1 so that the Ricci curvature equals -1 , i.e., $\partial^{2} \log g(z) / \partial z \overline{\partial z}=g(z)$.

Our point is to give a direct proof, which only uses the hyperbolic geometry of Riemann surfaces.

We consider a closed geodesic $\gamma$ on $X$ parameterized by $u(t)$ with $\|\dot{u}(t)\|=1$. Let the length of $\gamma$ be $\ell_{0}=2 \pi r_{0}$. The twist along $\gamma$ gives rise to a real, one parameter family of Riemann surfaces, in particular it gives rise to a real (analytic) curve in the appropriate Teichmüller space.

As local coordinate set we use an annulus $W=\left\{z \in \mathbb{C} ; r_{1}<|z|<r_{2}\right\}$ embedded into $X$, such that $\gamma$ corresponds to a circle $\left\{|z|=r_{0}\right\}$ for $r_{1}<r_{0}<r_{2}$, with parameterization $u(t)=r_{0} e^{\sqrt{-1} t / r_{0}}$ for $0 \leq t \leq \ell_{0}$.

For $\tau \in \mathbb{R}$ a family of holomorphic automorphisms of $W$ is given by the twist $z \mapsto e^{\sqrt{-1} \tau / r_{0}} z$. In the sense of Section 4 we interpret these as a family of vertical automorphisms with respect to an open covering of $X$ consisting of two elements. Now a differentiable trivialization (which is equal to the identity on the complement of $W$ ) is given by

$$
\zeta(z, \tau)=e^{\sqrt{-1} \frac{\tau}{r_{0}} h(r)} z,
$$

where $r=|z|$ and $h \in C^{\infty}\left(\left(r_{1}, r_{2}\right), \mathbb{R}\right)$ denotes a differentiable function which is identically zero near $r_{1}$, and identically equal to one near $r_{2}$. The value $\tau=\ell_{0}$ represents a Dehn twist.

We take a deformation theoretic standpoint, and we use in this context the shearing technique [WO2, Section 1]: A Beltrami differential will again stand for an infinitesimal deformation. If we replace in (11) a tangent vector $\partial / \partial s^{\kappa}$ by a real tangent vector $\partial / \partial \tau$, we obtain the image of its $(1,0)$-component under the Kodaira-Spencer map. The resulting Beltrami differential $\mu=\mu(z)(\partial / \partial z) \overline{d z}$ is identically zero on the complement of $W$. For $z \in W$ it follows immediately that

$$
\mu(z)=\frac{\sqrt{-1}}{2} \frac{z^{2}}{r r_{0}} h^{\prime}(r) .
$$


Now we can prove Theorem 1.2. The idea is to determine a Beltrami differential as a linear form on the cotangent space of the Teichmüller space.

The duality $H^{1}\left(X, \mathcal{T}_{X}\right) \times H^{0}\left(X, \mathcal{O}_{X}\left(2 K_{X}\right)\right) \rightarrow \mathbb{C}$ of tangent and cotangent spaces of $\mathcal{T}$ is given by

$$
\left(\mu(z) \frac{\partial}{\partial z} \overline{d z}, \varphi(z) d z^{2}\right) \longmapsto \int_{X} \mu(z) \varphi(z) d A,
$$

where $d A=\sqrt{-1} d z \wedge \overline{d z}$ is the Euclidean area element. Observe that $\mu$ need not be harmonic in this expression, in fact we get the same value for any function $h(r)$ with the above properties. The claim of the theorem follows from (15) in principle by Stokes' theorem - we can also let $h$ tend to the function $h_{0}$, which is defined to be equal to zero for $r<r_{0}$ and equal to one for $r \geq r_{0}$. Then $h^{\prime}(r)$ converges to a delta function, and integration over $r$ implies that for any quadratic holomorphic differential $\varphi$,

$$
(\mu, \varphi)=\frac{\sqrt{-1}}{2} r_{0} \int_{0}^{2 \pi} e^{2 \sqrt{-1} \vartheta} \varphi\left(r_{0} e^{\sqrt{-1} \vartheta}\right) d \vartheta
$$

Now the necessary reparameterization $\vartheta=t / r_{0}$ yields the claim of the theorem.

The reader might compare Theorem 1.2 with the monodromy integrals from [WO2], which we now recall. If $A$ is a hyperbolic element in $\operatorname{PSL}(2, \mathbb{R})$, lifted to $\hat{A} \in \mathrm{SL}(2, \mathbb{R})$, the monodromy integral is

$$
\mathcal{P}(\psi, \hat{A})=\frac{1}{2} \int_{s}^{A s} \psi \Omega_{\hat{A}}
$$

where $\psi$ is a quadratic holomorphic differential, and $\Omega_{\hat{A}}^{-1}$ a suitable Abelian differential. Then by [WO2, Lemma 4.2] the following formula holds for the twist tangent vector $\mu_{0}$ applied to a holomorphic quadratic differential

$$
\int_{\Delta} \mu_{0} \psi=-\sqrt{-1} \sigma_{\hat{A}} \eta(\hat{A})^{-1} \mathcal{P}(\psi, \hat{A}),
$$

where $\Delta$ is a fundamental domain, $\sigma_{\hat{A}}= \pm 1$, and $\eta(\hat{A})=\left((\operatorname{tr} \hat{A})^{2}-4\right)^{1 / 2}$.

\section{Computation of the Fenchel-Nielsen symplectic form}

The results of this section are valid for the Teichmüller space of (possibly weighted) punctured Riemann surfaces.

Combining our geodesic integral formulas (1) and (2) we immediately obtain a result, which was proved in the classical case by Wolpert ([WO2, Theorem 2.10]). 
Corollary 7.1. The vector fields $\partial / \partial \tau^{j}$ are Hamiltonian with respect to the Weil-Petersson form:

$$
\begin{aligned}
& \frac{\partial \ell^{j}}{\partial s^{\bar{\kappa}}}=-\sqrt{-1}\left\langle\frac{\partial}{\partial \tau^{j}}, \mu_{\kappa}\right\rangle, \\
& \frac{\partial \ell^{j}}{\partial s^{\kappa}}=\sqrt{-1}\left\langle\mu_{\kappa}, \frac{\partial}{\partial \tau^{j}}\right\rangle .
\end{aligned}
$$

Here, the Weil-Petersson inner product, which is defined initially on the complex tangent space, is extended in a natural way to the complexified real tangent space (which also contains the complex tangent vectors of type $(0,1)$ ).

Next, we prove the coincidence (3) of the Fenchel-Nielsen and Weil-Petersson symplectic forms using (1) and (2). We will give a short computational argument:

Proof of Theorem 1.3. We write $\omega^{\mathrm{WP}}$ in terms of Fenchel-Nielsen coordinates and begin with the contribution of the twist parameter involving $d \tau^{j} \wedge d \tau^{k}$. Since we are dealing with real forms, we need to show:

Claim. The real part

$$
\operatorname{Re}\left(\sqrt{-1}\left\langle\frac{\partial}{\partial s^{\alpha}}, \frac{\partial}{\partial s^{\beta}}\right) \frac{\partial s^{\alpha}}{\partial \tau^{j}} \frac{\partial s^{\bar{\beta}}}{\partial \tau^{k}}\right)
$$

vanishes, in particular, it is symmetric in $j$ and $k$.

Proof of the claim.

$$
\begin{aligned}
\sqrt{-1}\left\langle\frac{\partial}{\partial s^{\alpha}}, \frac{\partial}{\partial s^{\beta}}\right\rangle \frac{\partial s^{\alpha}}{\partial \tau^{j}} \frac{\partial s^{\bar{\beta}}}{\partial \tau^{k}} & =\sqrt{-1}\left\langle\frac{\partial}{\partial s^{\alpha}}, \frac{\partial s^{\beta}}{\partial \tau^{k}} \frac{\partial}{\partial s^{\beta}}\right) \frac{\partial s^{\alpha}}{\partial \tau^{j}} \\
& =\sqrt{-1}\left\langle\frac{\partial}{\partial s^{\alpha}}, \frac{\partial}{\partial \tau^{k}}\right) \frac{\partial s^{\alpha}}{\partial \tau^{j}} \quad \text { (type consideration) } \\
& \stackrel{(17)}{=} \frac{\partial \ell^{k}}{\partial s^{\alpha}} \cdot \frac{\partial s^{\alpha}}{\partial \tau^{j}} .
\end{aligned}
$$

The real part of this quantity equals

$$
\frac{1}{2} \frac{\partial \ell^{k}}{\partial \tau^{j}}=0
$$

We conclude the proof of Theorem 1.3. The vanishing of the coefficients of $d \ell^{k} \wedge d \ell^{m}$ is an immediate consequence of the "dual" equation (18), which may not be available at this point. However, we can revert here to Wolpert's purely (differential) geometric argument [WO4, Corollary 1.2], which involves orientation reversing (anti-holomorphic) symmetries. 
Finally, the coefficient of $d \tau^{j} \wedge d \ell^{m}$ in $\omega^{\mathrm{WP}}$ equals

$$
\begin{aligned}
& \sqrt{-1}\left(\left\langle\frac{\partial s^{\alpha}}{\partial \tau^{j}} \frac{\partial}{\partial s^{\alpha}}, \frac{\partial s^{\beta}}{\partial \ell^{m}} \frac{\partial}{\partial s^{\beta}}\right\rangle-\left\langle\frac{\partial s^{\alpha}}{\partial \ell^{m}} \frac{\partial}{\partial s^{\alpha}}, \frac{\partial s^{\beta}}{\partial \tau^{j}} \frac{\partial}{\partial s^{\beta}}\right\rangle\right) \\
& =\sqrt{-1}\left(\left\langle\frac{\partial}{\partial \tau^{j}}, \frac{\partial s^{\beta}}{\partial \ell^{m}} \frac{\partial}{\partial s^{\beta}}\right\rangle-\left\langle\frac{\partial s^{\alpha}}{\partial \ell^{m}} \frac{\partial}{\partial s^{\alpha}}, \frac{\partial}{\partial \tau^{j}}\right\rangle\right) \\
& \stackrel{(16),(17)}{=}-\left(\frac{\partial \ell^{j}}{\partial s^{\bar{\beta}}} \frac{\partial s^{\bar{\beta}}}{\partial \ell^{m}}+\frac{\partial \ell^{j}}{\partial s^{\alpha}} \frac{\partial s^{\alpha}}{\partial \ell^{m}}\right) \\
& =-\delta_{\ell m} .
\end{aligned}
$$

Now we obtain the following formula of Wolpert from the equality $\omega^{\mathrm{FN}}=\omega^{\mathrm{WP}}$ :

Corollary 7.2. The length coordinates $\ell^{j}$ give rise to Hamiltonian vector fields $\partial / \partial \ell^{j}$ :

$$
\frac{\partial \tau^{j}}{\partial s^{\alpha}}=-\sqrt{-1}\left\langle\frac{\partial}{\partial s^{\alpha}}, \frac{\partial}{\partial \ell^{j}}\right\rangle
$$

The above equation (17) also implies the following corollary, whose classical version is Theorem 2.11 from [WO2]: We first observe that (17) holds in a slightly more general situation. Namely, as usual, we pick local holomorphic coordinates $\left(s_{1}, \ldots, s_{N}\right)$ on the Teichmüller space near a point $s_{0}$ and denote by $\mu_{\alpha}$ the harmonic Beltrami differentials corresponding to the $\partial /\left.\partial s^{\alpha}\right|_{s_{0}}$. Then for any closed geodesic $\gamma$ on $X$ we have the length function $\ell(\gamma)$, and also $\partial / \partial \tau(\gamma)$ is defined at the given point $s_{0}$ without a choice of a system of Fenchel-Nielsen coordinates. Now (17) reads

$$
\frac{\partial \ell(\gamma)}{\partial s^{\alpha}}=\sqrt{-1}\left\langle\frac{\partial}{\partial s^{\alpha}}, \frac{\partial}{\partial \tau(\gamma)}\right\rangle .
$$

for all $\alpha=1, \ldots, N$.

Corollary 7.3. Let $\gamma$ and $\delta$ be closed geodesics on $X$. Then

$$
\frac{\partial \ell(\gamma)}{\partial \tau(\delta)}=-\frac{\partial \ell(\delta)}{\partial \tau(\gamma)} .
$$

Proof. We show that the left-hand side is skew symmetric in $\gamma$ and $\delta$.

$$
\begin{aligned}
& \frac{\partial \ell(\gamma)}{\partial \tau(\delta)}=2 \operatorname{Re}\left(\frac{\partial \ell(\gamma)}{\partial s^{\alpha}} \cdot \frac{\partial s^{\alpha}}{\partial \tau(\delta)}\right) \\
& \stackrel{(19)}{=} 2 \operatorname{Re}\left(\sqrt{-1} \frac{\partial s^{\alpha}}{\partial \tau(\delta)} \cdot\left\langle\frac{\partial}{\partial s^{\alpha}}, \frac{\partial}{\partial \tau(\gamma)}\right\rangle\right)
\end{aligned}
$$




$$
\begin{aligned}
& =2 \operatorname{Re}\left(\sqrt{-1} \frac{\partial s^{\alpha}}{\partial \tau(\delta)} \cdot\left\langle\frac{\partial}{\partial s^{\alpha}}, \frac{\partial s^{\beta}}{\partial \tau(\gamma)} \frac{\partial}{\partial s^{\beta}}\right\rangle\right) \quad \text { (type consideration) } \\
& =2 \operatorname{Re}\left(\sqrt{-1} G_{\alpha \bar{\beta}}^{\mathrm{WP}} \frac{\partial s^{\alpha}}{\partial \tau(\delta)} \frac{\partial s^{\bar{\beta}}}{\partial \tau(\gamma)}\right) \\
& =\sqrt{-1} G_{\alpha \bar{\beta}}^{\mathrm{WP}}\left(\frac{\partial s^{\alpha}}{\partial \tau(\delta)} \frac{\partial s^{\bar{\beta}}}{\partial \tau(\gamma)}-\frac{\partial s^{\alpha}}{\partial \tau(\gamma)} \frac{\partial s^{\bar{\beta}}}{\partial \tau(\delta)}\right) .
\end{aligned}
$$

\section{Weighted punctured Riemann surfaces and conical metrics}

Let $X$ be a compact Riemann surface of genus $p$ and $\boldsymbol{a}=\sum_{i=1}^{n} a_{i} p_{i}$ be an $\mathbb{R}$-divisor, with $0<a_{i} \leq 1$ for all $i$. Then $(X, \boldsymbol{a})$ is called a weighted punctured Riemann surface. We always assume that the degree of the $\mathbb{R}$-divisor $K_{(X, \boldsymbol{a})}:=K_{X}+\boldsymbol{a}$, is positive. It was shown by Hulin, McOwen and Troyanov [H-T], [McO], [T] that such surfaces possess unique hyperbolic conical metrics. These are characterized as follows:

(i) For $a_{j}=1$ the metric $g$ satisfies a Poincaré growth condition at $p_{j}$, i.e., $g=\frac{\rho(z)}{|z|^{2} \log ^{2}\left(|z|^{2}\right)}|d z|^{2}$ in some local holomorphic coordinate system, where $p_{j}$ corresponds to $z=0$, and $\rho$ is a continuous positive function.

(ii) For $0<a_{j}<1$ the metric is of the form $g=\frac{\rho(z)}{|z|^{a_{j}}}|d z|^{2}$, where $\rho$ is continuous.

In a holomorphic family, in particular regarding Teichmüller and moduli spaces, punctures are labeled. The complex structure of the resulting Teichmüller space $\mathcal{T}_{\boldsymbol{a}}$ of weighted punctured surfaces (with weights being fixed) can be identified with $\mathcal{T}_{p, n}$. However, weights give rise to a hierarchy of compactifications of the corresponding moduli spaces, which were extensively investigated by Hassett [HA].

On the other hand, hyperbolic conical metrics induce different Kähler structures on Teichmüller and moduli spaces. Generalized Weil-Petersson metrics were introduced and studied in [S-T1], [S-T2].

From the viewpoint of hyperbolic geometry, the Teichmüller space of weighted punctured Riemann surfaces were studied by Zhang in $[\mathrm{ZH}]$. Under the seemingly necessary assumption that the weights are between $1 / 2$ and 1 , Zhang showed the existence of Fenchel-Nielsen coordinates so that the Fenchel-Nielsen symplectic form $\omega^{\mathrm{FN}}$ becomes meaningful. The above range of weights contains the interesting weights of the form $1-1 / k$ that arise from finite quotients. Any pants decomposition of a Riemann surface with conical singularities with the above restriction avoids the punctures. So Theorems 1.1 and 1.2 are still valid.

Concerning the notion of harmonic Beltrami differentials, Hilbert space theory is not available in general. Instead we use equation (13) as a definition for the space 
$H^{1}(X, \boldsymbol{a})$ of such differentials on a weighted punctured Riemann surface $(X, \boldsymbol{a})$. In [S-T1] the existence of a duality

$$
H^{1}(X, \boldsymbol{a}) \times H^{0}\left(X, \mathcal{O}_{X}\left(2 K_{X}+D\right)\right) \rightarrow \mathbb{C},
$$

was shown, where $D=\sum_{i} p_{i}$, is the underlying divisor to $\boldsymbol{a}$ with weights one. Also (12) holds. The $L^{2}$ inner product on $H^{1}(X, \boldsymbol{a})$ induced by the conical hyperbolic metric gives rise to a generalized Weil-Petersson metric, which is of Kähler type [S-T1], [S-T2]. The statement and proof of the Theorem 1.3 can be carried over literally to this case. So all necessary conditions for the coincidence of $\omega^{\mathrm{FN}}$ and $\omega^{\mathrm{WP}}$ are satisfied for weighted punctured Riemann surfaces.

\section{References}

[E-M] D. B. A. Epstein and A. Marden, Convex hulls in hyperbolic space, a theorem of Sullivan, and measured pleated surfaces. In Analytical and geometric aspects of hyperbolic space, London Math. Soc. Lecture Note Ser. 111, Cambridge University Press, Cambridge 1987, 113-253. Zbl 0612.57010 MR 2235711 244, 245

[F-K] O. Forster and K. Knorr, Ein neuer Beweis des Satzes von Kodaira-Nirenberg-Spencer. Math. Z. 139 (1974), 257-291. Zbl 0279.32008 MR 0361161250

[HA] B. Hassett, Moduli space of weighted pointed stable curves. Adv. Math. 173 (2003), 316-352. Zbl 1072.14014 MR 1957831255

[H-T] D. Hulin and M. Troyanov, Prescribing curvature on open surfaces. Math. Ann. 293 (1992), 277-315. Zbl 0799.53047 MR 1166122255

[Mc] C. McMullen, Complex earthquakes and Teichmüller theory. J. Amer. Math. Soc. 11 (1998), 283-320. Zbl 0890.30031 MR 1478844244

[McO] R. McOwen, Prescribed curvature and singularities of conformal metrics on Riemann surfaces. J. Math. Anal. Appl. 177 (1993), 287-298. Zbl 0806.53040 MR 1224820255

[SCH1] G. Schumacher, Harmonic maps of the moduli space of compact Riemann surfaces. Math. Ann. 275 (1986), 455-466. Zbl 0601.32025 MR 0858289

[SCH2] G. Schumacher, The curvature of the Petersson-Weil metric on the moduli space of Kähler-Einstein manifolds. In Complex analysis and geometry, Univ. Ser. Math., Plenum Press, New York 1993, 339-354. Zbl MR1224820 MR 1211890247

[SCH3] G. Schumacher, The theory of Teichmüller spaces. A view towards moduli spaces of Kähler manifolds. In Several complex variables, VI, Encyclopedia Math. Sci. 69, Springer-Verlag, Berlin 1990, 251-310. Zbl 0735.32016 MR 1095093 247

[S-T1] G. Schumacher and S. Trapani, Variation of cone metrics on Riemann surfaces. J. Math. Anal. Appl. 311 (2005), 218-230. Zbl 1080.14033 MR 2165474 255, 256

[S-T2] G. Schumacher and S. Trapani, Weil-Petersson geometry for families of hyperbolic conical Riemann surfaces. Preprint 2008, arXiv:0809.0058. 255, 256 
[SIU] Y.-T. Siu, Curvature of the Weil-Petersson metric in the moduli space of compact Kähler-Einstein manifolds of negative first Chern class. In Contributions to several complex variables, Aspects Math. E9, Vieweg, Braunschweig 1986, 261-298. Zbl 0598.32020 MR 0859202 247

[T] M. Troyanov, Prescibing curvature on compact surfaces with conical singularities. Trans. Amer. Math. Soc. 324 (1991), 793-821. Zbl 0724.53023 MR 1005085255

[WE] A. Weil. Final report on contract AF 18(603)-57. In CEuvres scientifiques. Collected papers, Vol. II, Springer-Verlag, Berlin 1979, 390-395, 545-547. Zbl 0424.01028 MR 537935243

[WO1] S. Wolpert, An elementary formula for the Fenchel-Nielsen twist. Comment. Math. Helv. 56 (1981), 132-135. Zbl 0467.30036 MR 0615620244

[WO2] S. Wolpert, The Fenchel-Nielsen deformation. Ann. of Math. 115 (1982), 501-528. Zbl 0496.30039 MR 0657237 243, 244, 245, 251, 252, 254

[WO3] S. Wolpert, On the symplectic geometry of deformations of a hyperbolic surface. Ann. of Math. 117 (1983), 207-234. Zbl 0518.30040 MR 0690844243

[WO4] S. Wolpert, On the Weil-Petersson geometry of the moduli space of curves. Amer. J. Math. 107 (1985), 969-997. Zbl 0578.32039 MR 0796909 243, 253

[ZH] Y. Zhang, Hyperbolic cone-surfaces, generalized Markoff maps, Schottky groups and McShane's identity, Doctoral Thesis, Department of Mathematics, National University of Singapore, 2004. 244, 255

Received August 1, 2008

Reynir Axelsson, Department of Mathematics, University of Iceland, Dunhaga 5,

107 Reykjavík, Iceland

E-mail: reynir@raunvis.hi.is

Georg Schumacher, Fachbereich Mathematik und Informatik, Philipps-Universität Marburg, Lahnberge, Hans-Meerwein-Strasse, 35032 Marburg, Germany

E-mail: schumac@mathematik.uni-marburg.de 